\title{
Long-Term survival in a young adult with total artificial heart: A success story
}

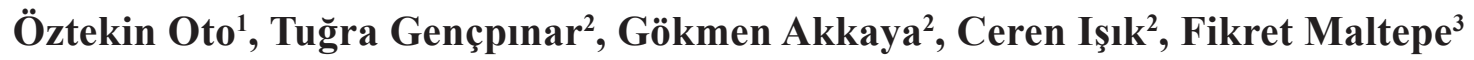

\footnotetext{
1) Dokuz Eylül University, Department of Cardiovascular Surgery, Prof. Dr., İzmir, Turkey

2) Dokuz Eylül University, Department of Cardiovascular Surgery, M.D., İzmir, Turkey

${ }^{3)}$ Dokuz Eylül University, Department of Anesthesiology and Reanimation, Prof. Dr., İzmir, Turkey
}

\begin{abstract}
We presented here the result of a successful surgical treatment of 16 years old male diagnosed as dilated cardiomyopathy with the history of siblings' early sudden death at the age of fifteen. The patient had to underwent total artificial heart implantation (TAH-T), (SynCardia) operation due to rapid biventricular detoriation and intracardiac trombus formation after his admittance to the ward. Due to lack of donor in the region he had to wait for long time with accurate anticoagulation and no serious complication during this long follow up period. He lived with TAH-T implantation both in the hospital and at home later was transplanted successfuly after 32 months. The patient is now activly working at a private company in NYHA Class 0 status. We have published this case due to its rarity in a young adult begins in childhood and longevity of the survival without any complication in TAH-T.
\end{abstract}

Keywords: Total artificial heart, SynCardia, Heart transplant, Dilated cardiomyopathy.

Oto Ö., Gençpınar T., Akkaya G., Işık C., Maltepe F. Long-Term survival in a young adult with total artificial heart: A success story. EJCM 2015; 03 (1): 7-10. DOI: 10.15511/ejcm.15.00107 


\section{Introduction}

Ventricular assist devices have been emerged for the pediatric population to mechanical circulatory support since late 1980s' as a bridge to heart transplantation (HTx).$^{[1]}$ Park and colleagues have demonstrated that the Total Artificial Heart (TAH-T) (SynCardia Systems Inc., Tuscon, AZ) has been approved for compassionate use by the Food and Drug Administration for patients with end-stage biventricular heart failure as a bridge to heart transplantation since 1985 and has had FDA approval since $2004 .^{[2]}$ In cases where total artificial heart (TAH-T) and ventricular assist devices (VAD) used as a bridge to heart transplantation mostly inhibits patients dying from end-stage bi-ventricular heart failure associated with ischemic or non-ischemic dilated cardiomyopathy. Beyond progressive chronic heart failure, the TAH-T has provided great efficacy in patients with acute irreversible heart failure. Altough common use of TAH-T in patients with heart failure since 80 's, usage in young adults and childhood age are rare. We present our experience with a young patient who had lived with a SynCardia TAH-T during 32 months for bridge to heart transplantation.

\section{Case Report}

16 years old- male had been admitted to our clinic with dilated cardiomyopathy. His background revealed that his elder brother had been diagnosed with same disease and died. He was suffering from exhaustion, exertional dyspnea and angina. His physical examination findings were fine crackles with lung auscultation and pretibial oedema $+++/+++$, based on these findings intravenous diuretic treatment was administered. In addition levosimendan treatment was initiated.

His echocardiogram resulted as left ventricule ejection fraction was $10-15 \%$, dilated cardiomyopathy, mild-severe mitrale regurgitation (accompanied with coaptation deficiency), mild-severe tricuspid regurgitation (accompanied with coaptation deficiency),

interventicular septum diameter: 0.8 , left ventricule diastolic diameter: 7.7, left ventricule systolic diameter: 7.4, left ventricule posterior wall diastolic diameter: 0.7 , right atrium: $7.5 \times 6.5 \mathrm{~cm}$, left atrium: $7.5 \times 7 \mathrm{~cm}$ and trombus formation was imaged in left atrial appendix.
Severe right ventricular failure has shown with low EF and high pulmonary arterial pressure (PAP) measured as $65 \mathrm{mmHg}$ and tricuspid annular plane systolic excursion (TAPSE) was scored $>18 \mathrm{~mm}$.

Cardiology- cardiovascular surgery multidisciplinary heart team decided on artificial heart implantation and immediate heart transplant for this particular patient. In 10.2012 the case underwent SynCardia total artificial heart implantation operation. He was extubated postop 1. day with no complications, the treatment carried on at cardiovascular surgery service unit. Acetylsalicylic acid 1x100 mg and warfarine dosage that arranged due to INR level were medicated daily. On account of prevention a possible thrombosis, the INR range was kept above 3. Following 32 months were complication-free. In the third year of our search for the donor heart we found a compatable one, unfortunately due to the short CPR story of the donor, we returned from the harvest empty-handed.

Finally in 06.2015 a suitable donor heart was found. Donor was a 23 year old male whom brain death had been occured, there was no story of CPR, with $2.5 \mathrm{mcg} /$ $\mathrm{kg} / \mathrm{min}$. dobutamine support, echocardiogram showed no contraction fault in his heart, 55\% left ventricule ejection fraction. After median sternotomy, we evaluated the donor heart. Neither contusion sign nor contraction fault were mentioned. The heart was harvested and transfered to our clinic in accuruate storage conditions. Before the operation, prednole and antibiotics administered to the patient. Patients were taken to the operation simultaneously, SynCardia total artificial heart was taken out. Transplant operation carried out with heterotropic heart implantation procedure.

In postop follow-up we detected over $100 \mathrm{cc} / \mathrm{h}$ bleeding from drainage tubes, because these findings indicated a possible hemorrhage, we immediately initiated revision surgery. The bleeding source was founded in the left atrium anastomose, and it had been controlled with resutures. After bleeding control completed, layers were closed in anatomical plan and patient was taken to the intensive care unit (ICU) again. $5 \mathrm{mcg} / \mathrm{kg} / \mathrm{min}$ dobutamine and $3 \mathrm{mcg} / \mathrm{kg} / \mathrm{min}$ dopamine infusions were administered. Blood gas values were controlled hourly and values were around normal range. Due to patients norological evaluation and awakeness, patient was 
extubated. Inotrope support was decreased and eventually stopped. Anti Tymocyte Globulin (ATG), prednole, tacrolimus and micofenolate mofetile treatments were initiated. White blood cell levels had dropped therefore ATG treatment was stopped and prednole dosage was decreased. Due to lack of C-reactive protein (CRP) increase, prednole treatment was stopped. Tacrolimus and micofenolate mofetile were administered at maintanance dosage. While admission he was suffered by fever and diarrea. After consulting the infectious disease department, we started variconasole, linezolide, cholistine, tigecycline combination as antibiotherapy. After the serologic tests resulted CMV antigenemia and high level of viral DNA load detected. The complications were considered to CMV infection thus valganciclovir therapy has started at dosage of $1000 \mathrm{mg} /$ day PO after induction therapy and revised due to viral load in follow-up.

Blood cultures, tracheal aspiration cultures, catheter cultures was examined in follow up regularly. Due to having no fever and diarrea anymore, we stopped antibiotheraphy after 15 days in addition there were no organism growth in any cultures and serologic tests. Later on patient had swelling and pain in his left leg, lower limb venous doppler ultrasound revealed subacute deep vein thrombosis in left leg, as treatment rivaroksaban was administered. In following days there were no other complications so patient was discharged.

At 04.2016, patient complained about left side pain and shortness of breath at $300 \mathrm{~m}$ walk. Lung x-ray showed thickened fissure, so we admitted the patient with prediagnosis of fissurite. Myocardial biopsy was performed to exclude rejection of donor. Biopsy results revealed no sign of rejection. Given the fact that he has a deep vein thrombosis story, thorax bt anjiography was performed to see if there is a clue of pulmonary embolism. Screening results showed no sign of thromboembolism. In follow-up symptoms regressed and patient was discharged.

\section{Discussion}

This case is rare because a 16 years old patient had waited with SynCardia total artıficial heart implantation among 32 long months for a heart transplant and bridge to transplantation (BTT) was successful.
Heart failure is as one of the top diagnoses for inpatients. The hospital admission mortality rates for heart failure are high with over $10 \%$ and over $20 \%$ mortal within 1 year of discharge. With limited numbers of solid organ donors for heart transplantation, one choice for this population is to use mechanical circulatory support devices.

Ventricular assist devices are widely accepted as a therapeutic option for bridge to transplant operation. ElBanayosy and colleagues have demonstrated that there are still limitations to the patient collective eligible for VAD placement, who might therefore benefit from the implantation of a total artificial heart. ${ }^{[1]}$ The SynCardia TAH-T is the only FDA-approved TAH-T in the world. ${ }^{[2]}$ The TAH-T is indicated for use as a BTT in patients at imminent risk of death from non-reversible bi-ventricular failure. ${ }^{[2]}$ The SynCardia is the only device that eliminates the symptoms. ${ }^{[2-5]}$ SynCardia TAH-T is the most effective and safe therapeutic system, providing a high cardiac output $(\sim 10 \mathrm{~L} / \mathrm{min})$ [6]. Nishimura et al. ${ }^{[7]}$ reported the importance of effective flow for the recovery of end-organ disfunction those who have nonreversible bi-ventricular failure with multi-system failure and also emphasized the necessity for a minimum cardiac index of $3.0 \mathrm{~L} / \mathrm{min} / \mathrm{m} 2$ for the recovery of preoperative end-organ dysfunction.

The TAH-T implantation is occurring at an ever increasing rate in the recent years. Slepian recommend that looking to the future a major unmet need remains in providing total heart support for children and small adults. ${ }^{[2]}$ The length of the blood-flow path is shorter and the inflow and outflow valves are larger than in any other bridge-totransplant device, resulting in greater blood flow at smaller pre-load. ${ }^{[3-4]}$ Such a device should be optimal for bridging transplant candidates who have biventricular failure and for whom all other therapies have failed. ${ }^{[4]}$

Dilated cardiomyopathy is the primary indication with an estimated 1.000 to 1.500 new pediatric cases diagnosed each year in the United States. ${ }^{[5]}$ The disease accounts for 65 percent of heart transplants in children 
11 to 17 years old. ${ }^{[6-8]}$ This group of patients is subject to the longest waiting-list mortality in Turkey.

Herein, the cardiology- cardiovascular surgery multidisciplinary team determined the SynCardia Heart was the only available choice for the patient because of exclusive conditions. The patient waited for an appropriate donor for cardiac transplantation nearly for 32 months at hospital. 16 years old- male was supported, rehabilitated and subsequently transplanted.

During device support, most mortality outcomes were related to pre-implant problems. Infection and stroke are rare events. ${ }^{[4]}$ Following the implantation, the patient experienced progressive subacute deep vein

\section{References}

1. El-Banayosy A, Arusoglu L, Morshuis M, et al. CardioWest total artificial heart: Bad Oeynhausen experience. Ann Thorac Surg 2005;80(2):548-52.

2. Slepian MJ, Alemu Y, Girdhar G, et al. The SynCardia(TM) total artificial heart: in vivo, in vitro, and computational modeling studies. J Biomech 2013,18;46(2):266-75.

3. Park SS, Sanders DB, Smith BP, et al. Total artificial heart in the pediatric patient with biventricular heart failure. Perfusion 2014;29(1):82-8.

4. Jack G, Richard G. Smith, Francisco A, et al. Total artificial heart bridge to transplantation: a 9-year experience with 62 patients. j.healun $2003 ; 07.024$.

5. Don Isaacs. Case Report on 14-Year-Old Pediatric Patient Bridged to thrombosis in left leg. During post-implant period, in case of a thrombosis, the INR range was kept above 3 .

In this article, we aimed to present our experience with a young patient who had waited with SynCardia total artificial heart implantation over years for a heart transplant and bridge to transplantation. Unfortunately, these patients on the waiting list can suffer from worsening of the clinical status or even die while waiting for the transplantation, and this occurs despite close follow-up and intensive medical therapy.

Funding: The authors received no financial support for the research or authorship of this article.

Transplant with SynCardia Total Artificial Heart. Perfusion 2013;11,1.

6. Kuçukaksu D, Pektok E, Arat N, et al. Total artificial heart for bridge to cardiac transplantation: first patient at home in Turkey. Turkish Journal of Thoracic and Cardiovascular Surgery 2013;21(4):1032-8.

7. Nishimura M, Nishimura $\mathrm{T}$, Ishikawa $\mathrm{M}$, et al. Importance of luxury flow for critically ill patients receiving a left ventricular assist system. J Artif Organs 2006;9:209-13.

8. Copeland JG, Smith RG, Arabia FA, et al. Comparison of the CardioWest total artificial heart, the novacor left ventricular assist system and the thoratec ventricular assist system in bridge to transplantation. Ann Thorac Surg 2001;71:S92-7.

Received: 23/12/2014

Accepted: $12 / 05 / 2015$

Published: 15/06/2015

Disclosure and conflicts of interest:

The authors declare no conflict of interest.

\section{Corresponding author:}

Dr. Tuğra Gençpınar

Mail: tugra01@yahoo.com 